\title{
Efficient collision detection for moving polyhedra
}

\author{
Elmar Schömer* Christian Thiel ${ }^{\dagger}$
}

April 25, 1995

\begin{abstract}
In this paper we consider the following problem: given two general polyhedra of complexity $n$, one of which is moving translationally or rotating about a fixed axis, determine the first collision (if any) between them. We present an algorithm with running time $O\left(n^{8 / 5+\epsilon}\right)$ for the case of translational movements and running time $O\left(n^{5 / 3+\epsilon}\right)$ for rotational movements, where $\epsilon$ is an arbitrary positive constant. This is the first known algorithm with sub-quadratic running time.
\end{abstract}

\footnotetext{
*Universität des Saarlandes, Fachbereich 14, Informatik, Im Stadtwald, D-66041 Saarbrücken, Germany. E-mail: schoemer@cs.uni-sb.de.

${ }^{\dagger}$ Max-Planck-Institut für Informatik, Im Stadtwald, D-66123 Saarbrücken, Germany. E-mail: thielompi-sb.mpg.de. This author was supported by the ESPRIT Basic Research Actions Program, under contract No. 7141 (project ALCOM II).
} 


\section{Introduction}

The demands on quality, security and higher production capacity in manufacturing increase the need for automation during the phase of product design. To find potential faults in the design as soon as possible one uses simulation programs: these predict the physical properties and reactions of the product and check whether particular prefabricated parts can be easily assembled. For the latter purpose, the simulation of assemblies and robots, efficient methods for collision detection are needed. In general collision detection is an essential prerequisite of simulations of mechanical series.

Regarding the significance of this problem we consider in our paper efficient algorithms for collision detection. It is known ([Bo79, CA84]) that in $\mathbb{R}^{3}$ a collision between a moving polyhedral object and a stationary obstacle is computable in time $O\left(n^{2}\right)$. Hereby $n$ denotes the complexity of the two objects, i.e. the number of vertices, edges and faces. We attempt to solve this problem in sub-quadratic time.

Our results are based on the following model:

- Objects are rigid bodies (polyhedra) in $\mathbb{R}^{3}$, their surfaces consists of planar faces with straight boundaries.

- An object may be moving translationally in an arbitrary direction or it may be rotating about an arbitrary axis.

These restrictions are based on the fact, that real objects can be easily modelled by polyhedra and every motion can be approximated by a sequence of translations and rotations. As model of computation we take the standard Real-RAM-model ([PS88]).

\subsection{Previous results}

There are (up to now) only efficient solutions for some special cases of translated polyhedra. Dobkin and Kirkpatrick demonstrate in [DK85, DK90] the efficiency of the hierarchical representation for solving distance problems between convex polyhedra: 11sing this data structure one can determine the collision between two convex polyhedra in time $O\left(\log ^{2} n\right)$, if only one of them is moving. If one of the objects is not convex an algorithm with running time $O(n \log n)$ is possible (for more details see [Sch94, DHKS90]). In his Ph.D. thesis the first author [SCH94] constructs an algorithm which computes the collision between a moving and a stationary iso-oriented polyhedron: if there are only constantly many $(c)$ possible directions of the edges then he is able to find a solution in time $O\left(c^{2} n \log ^{2} n\right)$. Also in this work the first sub-quadratic collision detection algorithm for two general polyhedra (one of which is translated and one is stationary) is developed. Before it was

only known how to decide in sub-quadratic time (see [PEL93]), whether two (stationary) general polyhedra intersect.

For the case of rotations there are no sub-quadratic algorithms known. Even the special case of two convex polyhedra, one of which is rotating has not been solved up to now. 
This particular problem was posed as an open question by Jack Snoeyink during the Third Dagstuhl Seminar on Computational geometry in March 1993:

Given two convex polyhedra $A, B$, and an axis of rotation, compute the smallest angle by which $B$ has to rotate to meet $A$. Can this be done in sub-quadratic time?

\subsection{New results}

In this paper we give the first sub-quadratic algorithms, which solve the collision problem between two general polyhedra, one of which is moving translationally or rotating about a fixed axis, whereas the other is stationary. In particular we get an upper bound of $O\left(n^{8 / 5+\epsilon}\right)$ for the translational movement and $O\left(n^{5 / 3+\epsilon}\right)$ for the rotational movement ${ }^{*}$.

\subsection{Outline}

The first collision between two polyhedra can either be a collision between a vertex of one polyhedron and a facet of the other or a collision between two edges. The former case is the simpler one and will be treated in the last part of the paper by plane sweep techniques (see section 6 ). The latter problem is the harder problem and we concentrate on it. We show how to preprocess the set of stationary segments, such that we can efficiently compute the first segment hit by a moving query segment. We proceed in three steps: In the first step we use the parametric search technique of Meggido (see [MEG83]) to reduce the problem of computing the first intersection during the motion to the problem of computing the total number of intersections during the motion. In the second step we show how to reduce the latter problem to a combination of halfspace- and simplex range searching problems; the key technique here is linearization, previously used in [AM92B]. In the third step we solve the range searching problems using known techniques of Kreveld and Matoušek. After that our general technique can be applied to the collision problem of line segments which move translationally or rotate about a fixed axis: in section 4 we will deduce the needed appropriate linearizations. Applying a recent result of Pellegrini [PeL93] we give an alternative solution for translational movement in section 5. Section 6 considers the collision problem for facets and vertices.

\section{General collision detection and parametric search}

\subsection{The problem}

Let $\mathcal{T}$ be a class of (topologically closed) geometric objects, i.e. closed subsets of $\mathbb{R}^{d}$, and let $\mathcal{S}$ be some set of $n$ objects in $\mathcal{T}$. Let $\mathcal{Q}$ be another class of (topologically closed) geometric objects in $\mathbb{R}^{d}$. Further let $\mathcal{M}$ be a set of admissible motions for the objects in $\mathcal{Q}$, i.e. in our case the set of all possible translations respectively rotations.

\footnotetext{
${ }^{*}$ Throughout this paper, $\epsilon$ denotes an arbitrary small positive constant.
} 
For an object $S$ of $\mathcal{T}$ and an object $Q \in \mathcal{Q}$, which moves according to a formula $\ell$, we denote the first time, such that $S$ is hit by $Q$, with $\phi(S, Q, \ell)$. If there is no such collision we set $\phi(S, Q, \ell)=\infty$. Abusing the notation slightly we use $\phi(S, Q, \ell)$ also for denoting the object containing the intersection point. Our goal is to build a data structure that, given a query object $Q \in \mathcal{Q}$ and the equation $\ell \in \mathcal{M}$ of a motion, computes quickly $\phi(\mathcal{S}, Q, \ell):=\min _{S \in \mathcal{S}} \phi(S, Q, \ell)$ together with a $S \in \mathcal{S}$ such that $\phi(S, Q, \ell)=\phi(\mathcal{S}, Q, \ell)$. We call this the on-line collision problem for $\mathcal{Q}$ with respect to $\mathcal{T}$. If we know $n$ queries in advance we consider the so-called batched collision problem for $\mathcal{Q}$ with respect to $\mathcal{T}$.

\subsection{The parametric search technique}

The parametric search technique (see [MEG83]) is a powerful tool for solving a variety of optimization problems efficiently. It can be described as follows: we consider a decision problem $\mathcal{P}(t)$ that receives as input $n$ data items and a real parameter $t$. Assume that $\mathcal{P}$ is monotone, meaning that if $\mathcal{P}\left(t_{0}\right)$ is true for some $t_{0}$, then $\mathcal{P}(t)$ is also true for all $t<t_{0}$. Our aim is to find the minimum value of $t$ for which $\mathcal{P}(t)$ is false. We denote this value by $t^{*}$.

Suppose we have an efficient algorithm $A_{s}$ that, given the $n$ data items and $t$, decides if $\mathcal{P}(t)$ is true or not, i.e. the algorithm $A_{s}$ can determine whether the given $t$ is equal to, smaller than, or larger than the desired value $t^{*}$ (we have $t \leq t^{*}$ iff $\mathcal{P}(t)$ is true). We call such a procedure an emptiness algorithm for $\mathcal{P}(t)$. The parametric search technique allows us to use that algorithm as a subroutine for solving the optimization problem, if the control flow of our emptiness algorithm $A_{s}$ is governed by comparisons, each of which involves testing the sign of some low-degree polynomials in $t$. The parametric search technique simulates $A_{s}$ generically on the unknown critical value $t^{*}$. Whenever $A_{s}$ reaches a branching operation, the comparison can be reduced to testing the sign of a suitable low-degree polynomial $f(t)$ at $t=t^{*}$. The algorithm computes the roots of this polynomial and checks each root to see whether it is less than or equal to $t^{*}$ by calling $A_{s}$. In this way, the algorithm identifies two successive roots between which $t^{*}$ must lie and thus determines the sign of $f\left(t^{*}\right)$. In this way we get an interval $I$ in which $t^{*}$ lies. As we proceed through the execution, each comparison that we resolve constrains $I$ further and we get a sequence of progressively smaller intervals each known to contain $t^{*}$. The generic simulation (since it is able to correctly resolve each comparison at each branching point) will run to completion and we are left with an interval $I$ that contains $t^{*}$. It can be shown that for any real number $r \in I, \mathcal{P}(r)$ is true. Therefore, $t^{*}$ must be the right endpoint of $I$.

Let $T_{s}$ and $C_{s}$ denote the running time and the number of comparisons made by algorithm $A_{s}$, respectively. Since $A_{s}$ makes at most $C_{s}$ comparisons during its execution, the entire simulation and, hence, the computation of $t^{*}$ take $O\left(C_{s} T_{s}\right)$ time. To speed up this algorithm, Megiddo replaces $A_{s}$ by a parallel algorithm $A_{p}$ that uses $P$ processors and runs in $T_{p}$ parallel time. At each parallel step, let $A_{p}$ make a maximum of $W_{p}$ independent comparisons. Then our algorithm simulates $A_{p}$ sequentially, again at the unknown value $t^{*}$. At each parallel step, we get at most $W_{p}$ low-degree polynomials in $t$. We compute the roots of all of them and do a binary search among them using repeated median finding to make the probes for $t^{*}$. For each probe, we run the sequential algorithm $A_{s}$. In this way, 
we get the correct sign of each polynomial in $t^{*}$, and our algorithm can simulate the next parallel step of $A_{p}$.

For the simulation of each parallel step, we spend $O\left(W_{p}\right)$ time for median finding. Hence, the entire simulation of this step takes time $O\left(W_{p}+T_{s} \log W_{p}\right)$. As a result, the entire algorithm computes $t^{*}$ in time $O\left(W_{p} T_{p}+T_{s} T_{p} \log W_{p}\right)$. Since $W_{p} \leq P$, the running time is bounded by $O\left(P T_{p}+T_{s} T_{p} \log P\right)$.

In order to apply Meggiddo's technique to our problem we need an algorithm $A_{s}$ that, given a query object $Q \in \mathcal{Q}$ and a motion $\ell \in \mathcal{M}$, decides whether the moving object intersects some objects of $\mathcal{S}$ within a given time period $[0, t]$. In our case this time period is represented by the length of a translation or by the angle of a rotation. We also assume that the algorithm can detect the case when exactly one object of $\mathcal{S}$ is intersected and that it can identify this object. Using this emptiness algorithm we can easily decide if a given time $t$ is less, equal or greater than $\phi(\mathcal{S}, Q, \ell)$ which allows us to apply Megiddo's parametric search technique.

\section{The emptiness algorithm}

Our strategy is to reduce the collision problem to a problem for other objects that do not move and then solve the latter by known techniques. We will proceed in two steps. Firstly we linearize the problem and construct a multilevel data structure for counting all collisions (respectively for testing, if there is any collision) within a given time interval. Then we modify this algorithm and get the emptiness algorithm needed for the parametric search technique.

In many applications one (complicated) query problem can be expressed as the combination of several other (easier) query problems.

A general notion for the composition of general query problems was introduced in [KREV92]:

Let $\mathcal{P}=\left\{p_{1}, p_{2}, \ldots p_{n}\right\}$ be a set of $n$ points in $\mathbb{R}^{d}$, let $\mathcal{R}$ denote the set of all simplices in $\mathbb{R}^{d}$, let $\mathcal{S}=\left\{s_{1}, \ldots, s_{n}\right\}$ be a set of $n$ objects, and let $\mathcal{Q}$ denote a set of queries on $\mathcal{S}$. The composed query problem $\left(\mathcal{S}^{\prime}, \mathcal{Q}^{\prime}\right)$ is defined as follows: $\mathcal{S}^{\prime}=\left\{\left(p_{i}, s_{i}\right) ; 1 \leq i \leq n\right\}$, $\mathcal{Q}^{\prime}=\mathcal{R} \times \mathcal{Q}$ and the answer set for a query $(R, Q) \in \mathcal{Q}^{\prime}$ is given by $\{(p, s) ;(p, s) \in$ $\mathcal{S}^{\prime}$ and $p \in R$ and $\left.s \in Q\right\}$. We also say that $\left(\mathcal{S}^{\prime}, \mathcal{Q}^{\prime}\right)$ is obtained from $(\mathcal{S}, \mathcal{Q})$ by simplex composition.

Simplices in $d$-space are the intersection of at most $d+1$ many halfspaces. Therefore we can w.l.o.g. consider simplex compositions where the simplices are halfspaces. In this case we also use the term halfspace composition. 


\subsection{General form of linearization}

In this section we introduce the concept of linearization. It allows to translate a complicated test in some low dimensional space into a test in some higher dimensional space but involving only linear tests. Here we want to test whether a moving object $Q$, whose location at time $\tau$ is described by $Q(\tau)$ intersects a stationary object $S$ in some time interval $[0 . . t]$. To find a linearization of this problem means to establish the equivalence

$$
[\exists \tau: 0<\tau<t, Q(\tau) \cap S \neq \emptyset] \Longleftrightarrow \bigvee_{i=1}^{\text {dis }} \bigwedge_{j=1}^{c o n}\left[\sum_{k=1}^{d i m} \delta_{k}^{i j}(Q, t) \zeta_{k}^{i j}(S) \bowtie 0\right]
$$

where $\bowtie \in\{<\rangle,,=, \leq, \geq\}$ for each innermost summation, dis, con, dim are positive constants, and $\delta_{k}^{i j}(Q, t)$ respectively $\zeta_{k}^{i j}(S)$ are rational functions of constant degree depending on the kind of motion. By $Q(\tau)$ we mean the location of $Q$ at time $\tau$.

Having such a linearization we map the objects $S \in \mathcal{S}$ into the points $p^{i j}:=$ $\left(\zeta_{1}^{i j}(S), \zeta_{2}^{i j}(S), \ldots, \zeta_{\operatorname{dim}}^{i j}(S)\right)$ in $\mathbb{R}^{\operatorname{dim}}$ and the query object $Q$ into the hyperplanes $h^{i j}:=\left(\delta_{1}^{i j}(Q, t), \delta_{2}^{i j}(Q, t), \ldots, \delta_{d i m}^{i j}(Q, t)\right)$ in the same space. Then we can think of any $\sum_{k=1}^{\operatorname{dim}} \delta_{k}^{i j}(Q, t) \zeta_{k}^{i j}(S) \bowtie 0$ as the condition, that (depending on $\bowtie$ ) the point $p^{i j}$ lies on the hyperplane $h^{i j}$ respectively in a halfspace bounded by $h^{i j}$. Because each conjunction of (1) can be interpreted as the composition of con halfspace range searching problems we can find the objects in $\mathcal{S}$ satisfying a particular conjunction by applying halfspace composition con times. The disjunctions of (1) correspond to the union of ranges. By rewriting the defining formula, we can assume that these are disjoint unions: a formula $A \vee B$ can be rewritten as $A \vee(B \wedge \neg A)$. Now for counting all objects hit by the moving query object we can just sum up the solutions of the dis $=O(1)$ composed problems (defined by the conjunctions).

In section 4 we deduce the linearization for the collision problem between a set of moving line segments (all moving in the same direction or all rotating about the same axis) and a set of stationary segments in $\mathbb{R}^{3}$. There we get $\operatorname{dim}=5$.

\subsection{The data structure}

In his Ph.D. thesis [Krev92] Marc van Kreveld investigated efficient solutions for simplex composition of query problems:

Theorem 1 ([Krev92]) Let $\mathcal{P}$ be a set of $n$ points in dim-space, and let $\mathcal{S}$ be a set of $n$ objects in correspondence with $\mathcal{P}$. Let $T$ be a data structure on $\mathcal{S}$ having building time $p(n)$, size $f(n)$ and query time $g(n)$. For an arbitrary small constant $\epsilon>0$, the application of simplex composition on $\mathcal{P}$ to $T$ results in a data structure $D$ of

1. size $O\left(n^{\epsilon}\left(n^{\operatorname{dim}}+f(n)\right)\right)$ and query time $O(g(n)+\log n)$, or

2. size $O(n+f(n))$ and query time $O\left(n^{\epsilon}\left(n^{1-1 / \operatorname{dim}}+g(n)\right)\right)$, or 
3. building time $O\left(m^{\epsilon}(m+p(n))\right)$, size $O\left(m^{\epsilon}(m+f(n))\right.$ and query time $O\left(n^{\epsilon}(g(n)+\right.$ $\left.n / m^{1 / \text { dim }}\right)$ ) for every fixed $m$ such that $n \leq m \leq n^{\text {dim }}$,

assuming that $f(n) / n$ is non-decreasing and $g(n) / n$ is non-increasing. Reporting takes additional $O(K)$ time if there are $K$ answers.

Remark: If there is a parallel query algorithm for $T$ running in time $t(n)$ using $P(n)$ processors then the query algorithm for the last data structure can be parallelized such that it runs in $O(t(n)+g(n)+\log n)$ parallel steps using $O\left(n^{\epsilon}\left(P(n)+n / m^{1 / d i m}\right)\right)$ processors, assuming that $P(n) / n$ is non-decreasing and $t(n) / n$ is non-increasing.

In our case we apply con $=O(1)$ halfspace compositions starting with a halfspace range searching problem. The resource bounds for halfspace range searching structures in dimspace are (see [MAT93])

- size and preprocessing time $O\left(n^{\text {dim }}\right)$, query time $O(\log n)$, or

- $O(m)$ space, preprocessing time $O\left(n^{1+\epsilon}+m(\log n)^{\epsilon}\right)$ and $O\left(\frac{n}{m^{1 / d i m}} \log ^{1-1 / d i m}(m / n)\right)$ query time for a parameter $n \leq m<n^{d i m}$.

Using these bounds Theorem 1 leads to a data structure with building time and size $O\left(m^{1+\epsilon}\right)$, which can count all objects in $S$ satisfying a particular conjunction of (1) in query time $T_{s}:=O\left(\frac{n^{1+\epsilon}}{m^{1 / d i m}}\right)$, for every fixed $m$ such that $n \leq m \leq n^{d i m}$. We can parallelize that query algorithm such that it runs in $T_{p}:=O($ polylog $n)$ parallel time with $P:=O\left(\frac{n^{1+\epsilon}}{m^{1 / d i m}}\right)$ processors. Using the parametric search technique this gives us the first time $t^{*}$ of any collision in time $O\left(P T_{p}+T_{s} T_{p} \log P\right)=O\left(\frac{n^{1+\epsilon}}{m^{1 / d i m}}\right)$. To get the first hit object we start the corresponding reporting algorithm satisfying the same resource bounds.

Theorem 2 The on-line collision problem with linearization (1) can be solved with a data structure of size and preprocessing time $O\left(m^{1+\epsilon}\right)$ and query time $O\left(\frac{n^{1+\epsilon}}{m^{1 / d i m}}\right)$, for every fixed $m$ such that $n \leq m \leq n^{\text {dim }}$.

Assume we have $n$ moving objects $Q \in \mathcal{Q}$ instead of only one and we want to determine the first collision between any pair $Q, S$, for $Q \in \mathcal{Q}$ and $S \in \mathcal{S}$. We apply the solution to the on-line problem and query the data structure of Theorem 2 with each moving element. This gives us a list of $n$ candidates in which we can find the first collision in time $O(n)$.

Using this approach we need $O\left(m^{1+\epsilon}\right)$ preprocessing time and $n \times O\left(\frac{n^{1+\epsilon}}{m^{1 / d i m}}\right)$ query time. To find the best time bound we have to minimize the function

$$
t(m)=c_{1} m^{1+\epsilon}+c_{2} \frac{n^{2+\epsilon}}{m^{1 / d i m}}
$$


where $c_{1}, c_{2}$ are the $O$-constants of the resource bounds. The value $t$ achieves its minimum for $m$ satisfying

$$
c_{1} m^{1+\epsilon}=c_{2} \frac{n^{2+\epsilon}}{m^{1 / d i m}} \text { i. e. } m=\left(\frac{c_{2}}{c_{1}}\right)^{\frac{d i m}{d i m \epsilon+d i m+1}} n^{\frac{(2+\epsilon) \operatorname{dim}}{d i m \epsilon+d i m+1}}=n^{\frac{2 \operatorname{dim}}{d \imath m+1}+\delta} .
$$

This proves the following result.

Corollary 3 Given a subset $S$ of $n$ objects from $\mathcal{S}$ and a set $Q$ of $n$ moving objects from $\mathcal{Q}$. Assume that there is a linearization of the collision problem for $\mathcal{Q}$ with respect to $\mathcal{T}$ in the form of (1). Then we can find in $O\left(n^{\frac{2 d i m}{d i m+1}+\epsilon}\right)$ time and space the first collision between any elements of $Q$ and $S$.

Corollary 4 Given two polyhedra of complexity n, one of which is moving translationally respectively is rotating about a fixed axis. The first collision between any two edges of them can be computed in time $O\left(n^{8 / 5+\epsilon}\right)$ respectively $O\left(n^{5 / 3+\epsilon}\right)$.

Remark: We can get a less efficient sub-quadratic algorithm in a simpler way. The data structure of polynomial size and logarithmic query time for halfspace range searching (first case of Theorem 1) leads to a data structure with building time and size $O\left(n^{\operatorname{dim}+\epsilon}\right)$, which can count all objects in $S$ satisfying a particular conjunction of (1) in query time $T_{s}:=O(\log n)$. Using the parametric search technique we modify the query algorithm such that it solves efficiently the on-line collision problem, i.e. it can determine the first collision of one query element in time $O\left(\log ^{2} n\right)$.

This is the basis for a sub-quadratic solution of the batched collision problem: If we want to compute the first collision between $n$ moving objects from $\mathcal{Q}$ and the $n$ stationary elements in $\mathcal{S}$ in sub-quadratic time we cannot directly use this data structure which requires at least time $O\left(n^{\operatorname{dim}+\epsilon}\right)$ for preprocessing. Instead we subdivide the set $S$ in $k$ subsets of (nearly) equal size and build for each subproblem the above data structure. This procedure needs preprocessing time $O\left(k(n / k)^{d i m+\epsilon}\right)$. Clearly we have to query each subproblem with each moving object. Therefore we have query time $O\left(n k \log ^{2}(n / k)\right)$. If we choose $k=n^{1-\frac{1}{d i m}} \geq 1$ we get a total time amount of $O\left(n^{2-\frac{1}{d i m}+\delta}\right)$, where $\delta$ denotes an arbitrary small positive constant.

\section{Collision of translationally or rotationally moving line segments}

\section{Formulation of the problem:}

Given: Two line segments $l_{a b}$ and $l_{c d}$ with endpoints $\mathbf{a}, \mathbf{b}$ and $\mathbf{c}, \mathbf{d}$. The line segment $l_{a b}(\tau)$ performs a translation in the direction of the positive $x_{3}$-axis or a counterclockwise rotation about the $x_{3}$-axis, from time $\tau=0$ to $\tau=t$.

Wanted: Linear conditions to describe the fact that there is a time $\tau, 0<$ $\tau<t$, such that $l_{a b}(\tau)$ and $l_{c d}$ intersect. 
In this section we show the following result:

For a translational as well as for a rotational motion there exist natural numbers dis, con, dim, so that the following holds:

$$
\left[\exists \tau: 0<\tau<t, l_{a b}(\tau) \cap l_{c d} \neq \emptyset\right] \Longleftrightarrow \bigvee_{i=1}^{\text {dis }} \bigwedge_{j=1}^{c o n}\left[\sum_{k=1}^{d i m} \zeta_{k}^{i j}(\mathbf{c}, \mathbf{d}) \delta_{k}^{i j}(\mathbf{a}, \mathbf{b}, t) \lessgtr 0\right],
$$

where $\zeta_{k}^{i j}(\mathbf{c}, \mathbf{d})$ is a polynomial in the coordinates of $\mathbf{c}$ and $\mathbf{d}$ and $\delta_{k}^{i j}(\mathbf{a}, \mathbf{b}, t)$ is a polynomial in $t$ and the coordinates of $\mathbf{a}$ and $\mathbf{b}$. These polynomials depend on the kind of motion.

Let $L_{a b}$ and $L_{c d}$ be the lines that contain the segments $l_{a b}$ and $l_{c d}$ respectively. Let $T=\left\{\tau \mid L_{a b}(\tau) \cap L_{c d} \neq \emptyset\right\}$. Then

$$
\left[\exists \tau: 0<\tau<t, l_{a b}(\tau) \cap l_{c d} \neq \emptyset\right] \Longleftrightarrow\left[\exists \tau \in T: 0<\tau<t \wedge l_{a b}(\tau) \cap l_{c d} \neq \emptyset\right] .
$$

\subsection{Plücker coordinates for lines in $\mathbb{R}^{3}$}

If $L_{a b} \cap L_{c d} \neq \emptyset$, then all four points $\mathbf{a}, \mathbf{b}, \mathbf{c}, \mathbf{d}$ lie in a plane. In homogeneous coordinates this fact can be expressed by the equation:

$$
\operatorname{det}\left[\begin{array}{cccc}
a_{0} & a_{1} & a_{2} & a_{3} \\
b_{0} & b_{1} & b_{2} & b_{3} \\
c_{0} & c_{1} & c_{2} & c_{3} \\
d_{0} & d_{1} & d_{2} & d_{3}
\end{array}\right]=0
$$

Expansion of this $4 \times 4$ determinant according to the $2 \times 2$ minors of the submatrix formed by the coordinates of the points $\mathbf{a}$ and $\mathbf{b}$ and the minors of the submatrix formed by the points $\mathbf{c}$ and $\mathbf{d}$ yields the following homogeneous equation:

$$
\begin{aligned}
& \gamma_{23} \alpha_{01}+\gamma_{31} \alpha_{02}+\gamma_{12} \alpha_{03}+\gamma_{03} \alpha_{12}+\gamma_{01} \alpha_{23}+\gamma_{02} \alpha_{31}=0 \\
& \text { with } \alpha_{i j}=a_{i} b_{j}-a_{j} b_{i} \text { and } \gamma_{i j}=c_{i} d_{j}-c_{j} d_{i} .
\end{aligned}
$$

For the sequel it is convenient to assume that our lines are oriented from the lower to the higher end point, i.e. $a_{3} \leq b_{3}$ and $c_{3} \leq d_{3}$ and hence $\alpha_{03} \geq 0$ and $\gamma_{03} \geq 0$. Moreover we restrict ourselves to the case $\alpha_{03}>0$ and $\gamma_{03}>0$, the other cases being simpler.

The Pliicker coordinates $\alpha_{i j}$ (and the Pliicker coefficients $\gamma_{i j}$ ) are not independent. They fulfill the equations

$$
\begin{aligned}
& \alpha_{01} \alpha_{23}+\alpha_{02} \alpha_{31}+\alpha_{03} \alpha_{12}=0 \\
& \gamma_{01} \gamma_{23}+\gamma_{02} \gamma_{31}+\gamma_{03} \gamma_{12}=0 .
\end{aligned}
$$

With the help of the bilinear equation (2) one can interpret the collision of the two lines $L_{a b}$ and $L_{c d}$ in $\mathbb{R}^{3}$ as a collision of a point $\mathbf{p}_{a b}$ with a hyperplane $H_{c d}$ in $\mathbb{R}^{6}$, where $\mathbf{p}_{a b}$ and $H_{c d}$ are given by:

$$
\begin{gathered}
H_{c d}: \gamma_{23} \xi_{1}+\gamma_{31} \xi_{2}+\gamma_{12} \xi_{3}+\gamma_{03} \xi_{4}+\gamma_{01} \xi_{5}+\gamma_{02} \xi_{6}=0 \\
\mathbf{p}_{a b}:\left(\xi_{1}, \xi_{2}, \xi_{3}, \xi_{4}, \xi_{5}, \xi_{6}\right)^{T}=\left(\alpha_{01}, \alpha_{02}, \alpha_{03}, \alpha_{12}, \alpha_{23}, \alpha_{31}\right)^{T}
\end{gathered}
$$




\subsection{Collision times for translationally moving lines}

In this subsection we compute the possible times of a collision between a translationally moving line $L_{a b}$ and a stationary line $L_{c d}$.

The translation of the line $L_{a b}(\tau)$ appears in Plïcker space as a corresponding motion of the point $\mathbf{p}_{a b}(\tau)$. Its Pliicker coordinates are obtained as the $2 \times 2$ minors of the following matrix:

$$
\begin{gathered}
{\left[\begin{array}{cccc}
a_{0} & a_{1} & a_{2} & a_{3}+\tau a_{0} \\
b_{0} & b_{1} & b_{2} & b_{3}+\tau b_{0}
\end{array}\right]} \\
\mathbf{p}_{a b}(\tau)=\left(\alpha_{01}, \alpha_{02}, \alpha_{03}, \alpha_{12}, \alpha_{23}-\tau \alpha_{02}, \alpha_{31}+\tau \alpha_{01}\right)
\end{gathered}
$$

Substituting these coordinates in the plane equation $H_{c d}$ we obtain:

$$
\begin{aligned}
& u_{1} \tau+u_{0}=0 \text { where } \\
u_{1}= & \gamma_{02} \alpha_{01}-\gamma_{01} \alpha_{02} \\
u_{0}= & \gamma_{23} \alpha_{01}+\gamma_{31} \alpha_{02}+\gamma_{12} \alpha_{03}+\gamma_{03} \alpha_{12}+\gamma_{01} \alpha_{23}+\gamma_{02} \alpha_{31} .
\end{aligned}
$$

In the general case, when the projections $\bar{L}_{a b}$ and $\bar{L}_{c d}$ of the lines onto the $x_{1} x_{2}$-plane are not parallel, we get $u_{1} \neq 0$ and therefore

$$
\tau_{0}=-\frac{u_{0}}{u_{1}}
$$

Otherwise, if $u_{1}=0$, a collision can only occur if $u_{0}=0$ and $\bar{L}_{a b}=\bar{L}_{c d}$. These degenerate cases will be treated in the appendix A.2. As far as the collision test for polyhedra is concerned these cases can be ignored, because they are detected during the collison test of vertices and facets (see section 6).

\subsection{Conditions for the collision of translationally moving lines}

We want to derive linear expressions, which only depend on the coordinates of $\mathbf{a}$ and $\mathbf{b}$, for the predicate $\left[0<\tau_{0}<t\right]$. We have the equivalence

$$
\begin{aligned}
& {\left[0<\tau_{0}<t\right]} \\
& \Longleftrightarrow \begin{array}{l}
{\left[u_{1}>0\right] \wedge\left[u_{0}<0\right] \wedge\left[t u_{1}+u_{0}>0\right]} \\
\vee\left[u_{1}<0\right] \wedge\left[u_{0}>0\right] \wedge\left[t u_{1}+u_{0}<0\right]
\end{array}
\end{aligned}
$$

where the term $t u_{1}+u_{0}$ can be written in linearized form as follows:

$$
t u_{1}+u_{0}=\gamma_{02}\left(t \alpha_{01}+\alpha_{31}\right)+\gamma_{01}\left(-t \alpha_{02}+\alpha_{23}\right)+\gamma_{03} \alpha_{12}+\gamma_{12} \alpha_{03}+\gamma_{23} \alpha_{01}+\gamma_{31} \alpha_{02}
$$

This gives a linearized form for the predicate $\left[0<\tau_{0}<t\right]$ of dimension 6 . 


\subsection{Conditions for the collision of translationally moving line segments}

\section{Formulation of the problem:}

Given: Two line segments $l_{a b}$ and $l_{c d}$ with endpoints $\mathbf{a}, \mathbf{b}$ and $\mathbf{c}, \mathbf{d}$. Assume that during a translation of $l_{a b}$ in the direction of the positive $x_{3}$-axis the condition $\left[L_{a b}\left(\tau_{0}\right) \cap L_{c d} \neq \emptyset\right]$ is valid at time $\tau_{0}$.

Wanted: Linear conditions to describe the fact, that $\left[l_{a b}\left(\tau_{0}\right) \cap l_{c d} \neq \emptyset\right]$.

We use the following relation in order to answer the question, whether the line segments really intersect, in case the corresponding lines collide:

$$
\left[l_{a b}\left(\tau_{0}\right) \cap l_{c d} \neq \emptyset\right] \Longleftrightarrow\left[\bar{l}_{a b}\left(\tau_{0}\right) \cap \bar{l}_{c d} \neq \emptyset\right]
$$

With $\bar{l}_{a b}\left(\tau_{0}\right)$ and $\bar{l}_{c d}$ we denote the projection of the two line segments onto the $x_{1} x_{2}$-plane. Note that $\bar{l}_{a b}\left(\tau_{0}\right)=\bar{l}_{a b}$ because $l_{a b}$ is moving in the positive $x_{3}$-direction.

\section{Projection of the line segments into the $x_{1} x_{2}$-plane}

We project the line segments $l_{a b}$ and $l_{c d}$ onto the $x_{1} x_{2}$-plane.

$$
\begin{aligned}
& \bar{l}_{a b}: \quad \overline{\mathbf{x}}=\overline{\mathbf{a}}+\lambda(\overline{\mathbf{b}}-\overline{\mathbf{a}}), \quad \text { where } \quad 0<\lambda<1 \\
& \bar{l}_{c d}: \quad \overline{\mathbf{X}}=\overline{\mathbf{c}}+\mu(\overline{\mathbf{d}}-\overline{\mathbf{c}}), \quad \text { where } \quad 0<\mu<1
\end{aligned}
$$

Then

$$
\begin{aligned}
& {\left[\bar{l}_{a b} \cap \bar{l}_{c d} \neq \emptyset\right] \Longleftrightarrow \quad\left(\left[\overline{\mathbf{c}} \text { left of } \bar{L}_{a b}\right] \wedge\left[\overline{\mathbf{d}} \text { right of } \bar{L}_{a b}\right]\right.} \\
& \left.\wedge\left[\begin{array}{lll}
\overline{\mathbf{a}} & \text { right of } & \bar{L}_{c d}
\end{array}\right] \wedge\left[\begin{array}{lll}
\overline{\mathbf{b}} & \text { left of } & \bar{L}_{c d}
\end{array}\right]\right) \\
& \text { v } \\
& \left(\left[\overline{\mathbf{c}} \text { right of } \bar{L}_{a b}\right] \wedge\left[\overline{\mathbf{d}} \text { left of } \bar{L}_{a b}\right]\right.
\end{aligned}
$$

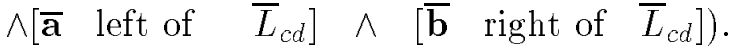

The point $\overline{\mathbf{c}}$ lies to the left/right of the orientated line $\bar{L}_{a b}$ iff the following is true:

$$
\begin{aligned}
& \left((\mathbf{b}-\mathbf{a}) \times(\mathbf{c}-\mathbf{a})_{3} \lessgtr 0\right. \\
\Longleftrightarrow & (\mathbf{a} \times \mathbf{b})_{3}+(\mathbf{b} \times \mathbf{c})_{3}+(\mathbf{c} \times \mathbf{a})_{3} \lessgtr 0 \\
\Longleftrightarrow & a_{1} b_{2}-a_{2} b_{1}+c_{2} b_{1}-c_{1} b_{2}+c_{1} a_{2}-c_{2} a_{1} \lessgtr 0 .
\end{aligned}
$$

Therefore

$$
\begin{aligned}
{\left[\bar{l}_{a b} \cap \bar{l}_{c d} \neq \emptyset\right] \Longleftrightarrow } & \left(\left[a_{1} b_{2}-a_{2} b_{1}+c_{2} b_{1}-c_{1} b_{2}+c_{1} a_{2}-c_{2} a_{1}>0\right]\right. \\
& \wedge\left[a_{1} b_{2}-a_{2} b_{1}+d_{2} b_{1}-d_{1} b_{2}+d_{1} a_{2}-d_{2} a_{1}<0\right] \\
& \wedge\left[c_{1} d_{2}-c_{2} d_{1}+d_{1} a_{2}-d_{2} a_{1}+c_{2} a_{1}-c_{1} a_{2}<0\right] \\
& \left.\wedge\left[c_{1} d_{2}-c_{2} d_{1}+d_{1} b_{2}-d_{2} b_{1}+c_{2} b_{1}-c_{1} b_{2}>0\right]\right) \\
& \vee \\
& \left(\left[a_{1} b_{2}-a_{2} b_{1}+c_{2} b_{1}-c_{1} b_{2}+c_{1} a_{2}-c_{2} a_{1}<0\right]\right. \\
& \wedge\left[a_{1} b_{2}-a_{2} b_{1}+d_{2} b_{1}-d_{1} b_{2}+d_{1} a_{2}-d_{2} a_{1}>0\right] \\
& \wedge\left[c_{1} d_{2}-c_{2} d_{1}+d_{1} a_{2}-d_{2} a_{1}+c_{2} a_{1}-c_{1} a_{2}>0\right] \\
& \left.\wedge\left[c_{1} d_{2}-c_{2} d_{1}+d_{1} b_{2}-d_{2} b_{1}+c_{2} b_{1}-c_{1} b_{2}<0\right]\right) .
\end{aligned}
$$




\subsection{Collision times for rotating lines}

A counterclockwise rotation of the line $L_{a b}(\varphi)$ about the $x_{3}$-axis induces a corresponding motion of the point $\mathbf{p}_{a b}(\varphi)$ in Pliicker space. Its Pliicker coordinates are given by the $2 \times 2$ minors of the following matrix:

$$
\begin{aligned}
& \mathbf{p}_{a b}(\varphi)=\left.\begin{array}{cccc}
a_{0} & \cos \varphi a_{1}+\sin \varphi a_{2} & -\sin \varphi a_{1}+\cos \varphi a_{2} & a_{3} \\
b_{0} & \cos \varphi b_{1}+\sin \varphi b_{2} & -\sin \varphi b_{1}+\cos \varphi b_{2} & b_{3}
\end{array}\right] \\
&\left(\begin{array}{cc}
\cos \varphi \alpha_{01}+\sin \varphi \alpha_{02},-\sin \varphi \alpha_{01}+\cos \varphi \alpha_{02}, \alpha_{03}, \\
\alpha_{12}, \cos \varphi \alpha_{23}+\sin \varphi \alpha_{31},-\sin \varphi \alpha_{23}+\cos \varphi \alpha_{31}
\end{array}\right)
\end{aligned}
$$

Substituting these coordinates into the plane equation $H_{c d}$ results in:

$$
\begin{aligned}
& u_{2} \cos \varphi+u_{1} \sin \varphi+u_{0}=0 \text { where } \\
u_{2}= & \gamma_{23} \alpha_{01}+\gamma_{31} \alpha_{02}+\gamma_{01} \alpha_{23}+\gamma_{02} \alpha_{31} \\
u_{1}= & -\gamma_{31} \alpha_{01}+\gamma_{23} \alpha_{02}-\gamma_{02} \alpha_{23}+\gamma_{01} \alpha_{31} \\
u_{0}= & \gamma_{12} \alpha_{03}+\gamma_{03} \alpha_{12}
\end{aligned}
$$

The following parametric formulation

$$
\sin \varphi=\frac{2 \tau}{1+\tau^{2}}, \quad \cos \varphi=\frac{1-\tau^{2}}{1+\tau^{2}}, \quad \text { where } \quad \tau=\tan \frac{\varphi}{2}, 0<\varphi<\pi
$$

transforms equation (5) into a quadratic equation

$$
u_{2}^{\prime} \tau^{2}+u_{1}^{\prime} \tau+u_{0}^{\prime}=0, \quad \text { where } \quad u_{2}^{\prime}=u_{0}-u_{2}, \quad u_{1}^{\prime}=2 u_{1}, \quad u_{0}^{\prime}=u_{0}+u_{2}
$$

with the two roots:

$$
\tau_{1}=\frac{-u_{1}^{\prime}+\sqrt{u_{1}^{\prime 2}-4 u_{2}^{\prime} u_{0}^{\prime}}}{2 u_{2}^{\prime}}, \quad \tau_{2}=\frac{-u_{1}^{\prime}-\sqrt{u_{1}^{\prime 2}-4 u_{2}^{\prime} u_{0}^{\prime}}}{2 u_{2}^{\prime}}, \text { for } u_{2}^{\prime} \neq 0 .
$$

As expected there are in general two points in time where the two lines intersect. If $u_{2}^{\prime}=0$ and $u_{1}^{\prime} \neq 0$ there is one collision at time $-\frac{u_{0}^{\prime}}{u_{1}^{\prime}}$ and an other at $\infty$, which corresponds to a rotation angle of $\varphi=\pi$. The degenerate case $u_{2}^{\prime}=u_{1}^{\prime}=u_{0}^{\prime}=0$ occurs iff both lines $L_{a b}$ and $L_{c d}$ intersect the axis of rotation in the same point or if both are parallel to it or if both lie in the same plane perpendicular to the axis of rotation. In these cases we can proceed similar to the treatment of the degenerate cases of the translational motion.

\subsection{Conditions for the collision of rotating lines}

$\tau_{1}$ and $\tau_{2}$ are real numbers only if $\left[u_{1}^{\prime 2}-4 u_{2}^{\prime} u_{0}^{\prime} \geq 0\right]$. Under this precondition the predicates $\left[0<\tau_{i}<t\right]$ can be transformed as follows (see (13) in the appendix):

$$
\left[0<\tau_{1}<t\right]
$$




$$
\begin{array}{rr}
\Longleftrightarrow\left[u_{2}^{\prime}>0\right] \wedge\left(\left[u_{1}^{\prime}<0\right] \vee\left[u_{0}^{\prime}<0\right]\right) \wedge\left[2 t u_{2}^{\prime}+u_{1}^{\prime}>0\right] \wedge\left[t^{2} u_{2}^{\prime}+t u_{1}^{\prime}+u_{0}^{\prime}>0\right] \\
\vee\left[u_{2}^{\prime}<0\right] \wedge\left[u_{1}^{\prime}>0\right] \wedge\left[u_{0}^{\prime}<0\right] \wedge\left(\left[2 t u_{2}^{\prime}+u_{1}^{\prime}<0\right] \vee\left[t^{2} u_{2}^{\prime}+t u_{1}^{\prime}+u_{0}^{\prime}>0\right]\right) \\
\vee\left[u_{2}^{\prime}=0\right] \wedge\left[u_{1}^{\prime}>0\right] \wedge\left[u_{0}^{\prime}<0\right] \wedge
\end{array}
$$

and

$$
\begin{aligned}
& {\left[0<\tau_{2}<t\right]} \\
& \Longleftrightarrow \quad\left[u_{2}^{\prime}>0\right] \wedge\left[u_{1}^{\prime}<0\right] \wedge\left[u_{0}^{\prime}>0\right] \wedge\left(\left[2 t u_{2}^{\prime}+u_{1}^{\prime}>0\right] \vee\left[t^{2} u_{2}^{\prime}+t u_{1}^{\prime}+u_{0}^{\prime}<0\right]\right) \\
& \quad \vee\left[u_{2}^{\prime}<0\right] \wedge\left(\left[u_{1}^{\prime}>0\right] \vee\left[u_{0}^{\prime}>0\right]\right) \wedge\left[2 t u_{2}^{\prime}+u_{1}^{\prime}<0\right] \wedge\left[t^{2} u_{2}^{\prime}+t u_{1}^{\prime}+u_{0}^{\prime}<0\right] \\
& \quad \vee\left[u_{2}^{\prime}=0\right] \wedge\left[u_{1}^{\prime}<0\right] \wedge\left[u_{0}^{\prime}>0\right] \wedge
\end{aligned}
$$

We now derive the linear expressions for the various predicates. From equation (6) we obtain the following equations

$$
\begin{aligned}
2 t u_{2}^{\prime}+u_{1}^{\prime}= & 2 \gamma_{01}\left(-t \alpha_{23}+\alpha_{31}\right)+2 \gamma_{02}\left(-t \alpha_{31}-\alpha_{23}\right)+2 \gamma_{03}\left(t \alpha_{12}\right) \\
& +2 \gamma_{12}\left(t \alpha_{03}\right)+2 \gamma_{23}\left(-t \alpha_{01}+\alpha_{02}\right)+2 \gamma_{31}\left(-t \alpha_{02}-\alpha_{01}\right) \\
t^{2} u_{2}^{\prime}+t u_{1}^{\prime}+u_{0}^{\prime}= & \gamma_{01}\left(-t^{2} \alpha_{23}+2 t \alpha_{31}+\alpha_{23}\right)+\gamma_{02}\left(-t^{2} \alpha_{31}-2 t \alpha_{23}+\alpha_{31}\right) \\
& +\gamma_{03}\left(t^{2} \alpha_{12}+\alpha_{12}\right)+\gamma_{12}\left(t^{2} \alpha_{03}+\alpha_{03}\right)+\gamma_{23}\left(-t^{2} \alpha_{01}+2 t \alpha_{02}-\alpha_{01}\right) \\
& +\gamma_{31}\left(-t^{2} \alpha_{02}-2 t \alpha_{01}+\alpha_{02}\right) \\
u_{1}^{\prime 2}-4 u_{2}^{\prime} u_{0}^{\prime}= & \left(\gamma_{23}^{2}+\gamma_{31}^{2}\right)\left(\alpha_{01}^{2}+\alpha_{02}^{2}\right)+2\left(\gamma_{01} \gamma_{23}+\gamma_{02} \gamma_{31}\right)\left(\alpha_{01} \alpha_{23}+\alpha_{02} \alpha_{31}\right) \\
& -2\left(\gamma_{02} \gamma_{23}-\gamma_{01} \gamma_{31}\right)\left(\alpha_{02} \alpha_{23}-\alpha_{01} \alpha_{31}\right)-\gamma_{12} \alpha_{03}^{2}-2 \gamma_{03} \gamma_{12} \alpha_{03} \alpha_{12} \\
& -\gamma_{03} \alpha_{12}^{2}+\left(\gamma_{01}^{2}+\gamma_{02}^{2}\right)\left(\alpha_{23}^{2}+\alpha_{31}^{2}\right) \\
= & \left(\gamma_{23}^{2}+\gamma_{31}^{2}\right)\left(\alpha_{01}^{2}+\alpha_{02}^{2}\right)+\left(\gamma_{01}^{2}+\gamma_{02}^{2}\right)\left(\alpha_{23}^{2}+\alpha_{31}^{2}\right)-\gamma_{12}^{2}\left(\alpha_{03}^{2}\right) \\
& -2\left(\gamma_{02} \gamma_{23}-\gamma_{01} \gamma_{31}\right)\left(\alpha_{02} \alpha_{23}-\alpha_{01} \alpha_{31}\right)-\gamma_{03}^{2}\left(\alpha_{12}^{2}\right) .
\end{aligned}
$$

The last equality holds because of equation (3), since $\left(\gamma_{01} \gamma_{23}+\gamma_{02} \gamma_{31}\right)\left(\alpha_{01} \alpha_{23}+\alpha_{02} \alpha_{31}\right)=$ $\gamma_{03} \gamma_{12} \alpha_{03} \alpha_{12}$.

In each case the predicates $\left[t^{2} u_{2}^{\prime}+t u_{1}^{\prime}+u_{0}^{\prime} \lessgtr 0\right],\left[2 t u_{2}^{\prime}+u_{1}^{\prime} \lessgtr 0\right]$ and $\left[u_{1}^{\prime 2}-4 u_{2}^{\prime} u_{0}^{\prime} \geq 0\right]$ are linear in at most 6 expressions $\delta_{k}^{i j}(\mathbf{a}, \mathbf{b}, t)$, which are given as polynomials in the coordinates of $\mathbf{a}$ and $\mathbf{b}$ and the time parameter $t$. This means we have found a linearization of dimension 6 for the predicate $\left[0<\tau_{i}<t\right]$.

\subsection{Conditions for the collision of rotating line segments}

\section{Formulation of the problem:}

Given: Two line segments $l_{a b}$ and $l_{c d}$ with endpoints $\mathbf{a}, \mathbf{b}$ and $\mathbf{c}$, d. Assume that during the rotation of $l_{a b}(\tau)$ about the $x_{3}$-axis the predicate $\left[L_{a b}\left(\tau_{i}\right) \cap\right.$ $\left.L_{c d} \neq \emptyset\right]$ is valid at time $\tau_{i}$.

Wanted: Linear conditions to describe the fact, that $\left[l_{a b}\left(\tau_{i}\right) \cap l_{c d} \neq \emptyset\right]$.

We reduce the decision whether $\left[l_{a b}\left(\tau_{i}\right) \cap l_{c d} \neq \emptyset\right]$ to the calculation of the $x_{3}-$ coordinate $z$ of the intersection points of the corresponding lines and a test whether $z \in\left[\min \left\{a_{3}, b_{3}\right\}, \max \left\{a_{3}, b_{3}\right\}\right] \cap\left[\min \left\{c_{3}, d_{3}\right\}, \max \left\{c_{3}, d_{3}\right\}\right]$. For the calculation of the $x_{3}-$ coordinates of the possible intersection point we use cylindrical coordinates. 


\section{Representation of line segments in cylindrical coordinates}

If we represent the line segments

$$
\begin{aligned}
& l_{a b}: \quad \mathbf{x}=\mathbf{a}+\lambda(\mathbf{b}-\mathbf{a}), \quad \text { where } 0 \leq \lambda \leq 1, \\
& l_{c d}: \quad \mathbf{x}=\mathbf{c}+\mu(\mathbf{d}-\mathbf{c}), \quad \text { where } \quad 0 \leq \mu \leq 1 \text {, }
\end{aligned}
$$

in cylindrical coordinates $(r, \varphi, z)$, we can easily check, whether the line segment $l_{a b}$ can collide with the line segment $l_{c d}$ during a full rotation about the $x_{3}$-axis. During its rotation the line segment $l_{a b}$ generally describes a hyperboloid, whose projection into the $(r, z)$-plane of the cylindrical coordinate system yields a hyperbolic segment. The rotating line segment $l_{a b}$ can only collide with $l_{c d}$, if the two corresponding hyperbolic segments intersect in the $(r, z)$-plane. In order to compute this intersections we have to find the $(r, z)$-representation for each point in $\mathbb{R}^{3}$, which is given by its Cartesian coordinates $\left(x_{1}, x_{2}, x_{3}\right)$. We get

$$
\begin{aligned}
& z=x_{3}, \\
& r=\sqrt{x_{1}^{2}+x_{2}^{2}} .
\end{aligned}
$$

Since $x_{i}=a_{i}+\lambda\left(b_{i}-a_{i}\right)$ and $\lambda=\frac{z-a_{3}}{b_{3}-a_{3}}$, we have for $a_{3} \neq b_{3}$ :

$$
\begin{aligned}
r^{2} & =\left(a_{1}+\frac{z-a_{3}}{b_{3}-a_{3}}\left(b_{1}-a_{1}\right)\right)^{2}+\left(a_{2}+\frac{z-a_{3}}{b_{3}-a_{3}}\left(b_{2}-a_{2}\right)\right)^{2} \\
& =\frac{1}{\left(b_{3}-a_{3}\right)^{2}}\left(\left(a_{1} b_{3}-a_{3} b_{1}+z\left(b_{1}-a_{1}\right)\right)^{2}+\left(a_{2} b_{3}-a_{3} b_{2}+z\left(b_{2}-a_{2}\right)\right)^{2}\right) .
\end{aligned}
$$

We proceed with Pliicker coordinates and get:

$$
\begin{aligned}
& r^{2}=\frac{1}{\alpha_{03}^{2}}\left(\left(\alpha_{31}-z \alpha_{01}\right)^{2}+\left(\alpha_{23}+z \alpha_{02}\right)^{2}\right) \\
& =v_{2} z^{2}+v_{1} z+v_{0} \text {, } \\
& \text { where } v_{2}=\frac{\alpha_{01}^{2}+\alpha_{02}^{2}}{\alpha_{03}^{2}}, \quad v_{1}=2 \frac{\alpha_{02} \alpha_{23}-\alpha_{01} \alpha_{31}}{\alpha_{03}^{2}}, \quad v_{0}=\frac{\alpha_{23}^{2}+\alpha_{31}^{2}}{\alpha_{03}^{2}} \text {. }
\end{aligned}
$$

The question whether the line segment $l_{a b}$ collides with the stationary segment $l_{c d}$ while it is rotating about the $x_{3}$-axis can be answered by calculating the intersection between the following two parabolic segments:

$$
\begin{array}{llll}
r_{a b}^{2}(z)=v_{2} z^{2}+v_{1} z+v_{0} & \text { with } & a_{3} \leq z \leq b_{3}, \\
r_{c d}^{2}(z)=w_{2} z^{2}+w_{1} z+w_{0} & \text { with } & c_{3} \leq z \leq d_{3} .
\end{array}
$$

W.l.o.g. let the line segments be given, such that $a_{3} \leq b_{3}$ and $c_{3} \leq d_{3}$. The intersection points of the two parabola can be found as the roots of a quadratic equation:

$$
\begin{aligned}
& v_{2}^{\prime} z^{2}+v_{1}^{\prime} z+v_{0}^{\prime}=0 \quad \text { where } \quad v_{i}^{\prime}=v_{i}-w_{i}, \\
& z_{1}=\frac{-v_{1}^{\prime}+\sqrt{v_{1}^{\prime 2}-4 v_{2}^{\prime} v_{0}^{\prime}}}{2 v_{2}^{\prime}}, \quad z_{2}=\frac{-v_{1}^{\prime}-\sqrt{v_{1}^{\prime 2}-4 v_{2}^{\prime} v_{0}^{\prime}}}{2 v_{2}^{\prime}}, \quad \text { for } v_{2}^{\prime} \neq 0
\end{aligned}
$$

But a collision of the rotating line segment $l_{a b}$ with $l_{c d}$ exists only if the quadratic equation has real roots $\left(\left[v_{1}^{\prime 2}-4 v_{2}^{\prime} v_{0}^{\prime} \geq 0\right]\right)$ and these lie in the interval $\left[a_{3}, b_{3}\right] \cap\left[c_{3}, d_{3}\right]$. For $v_{2}^{\prime}=0$ 
and $v_{1}^{\prime} \neq 0$ we get one solution at $-\frac{v_{0}^{\prime}}{v_{1}^{\prime}}$ and an other at $\infty$. The case $v_{2}^{\prime}=v_{1}^{\prime}=v_{0}^{\prime}=0$ occurs iff $L_{c d}$ lies on the hyperboloid generated by the rotating line $L_{a b}$. In this case possible collisions can be ignored because they are detected when testing vertices against facets.

By using cylindrical coordinates we have succeeded in finding the $x_{3}$-coordinates of the possible intersection points of the rotating line $L_{a b}(\tau)$ with $L_{c d}$. But it remains open, to which $x_{3}$-coordinate the collision time $\tau_{i}$ corresponds. In section A.3 we show, that $z_{1}$ belongs to $\tau_{1}$ and $z_{2}$ to $\tau_{2}$, if we assume that $a_{3}<b_{3}$ and $c_{3}<d_{3}$. That is

$$
\left[l_{a b}\left(\tau_{i}\right) \cap l_{c d} \neq \emptyset\right] \Longleftrightarrow\left[z_{i}>a_{3}\right] \wedge\left[z_{i}<b_{3}\right] \wedge\left[z_{i}>c_{3}\right] \wedge\left[z_{i}<d_{3}\right]
$$

In the following we want to derive linearized conditions for the predicates $\left[z_{i}<Z\right]$ respectively $\left[z_{i}>Z\right]$ with the help of relations (13).

$$
\begin{aligned}
& {\left[z_{1}<Z\right] \Longleftrightarrow \quad\left[v_{2}^{\prime}>0\right] \wedge\left[2 Z v_{2}^{\prime}+v_{1}^{\prime}>0\right] \wedge\left[Z^{2} v_{2}^{\prime}+Z v_{1}^{\prime}+v_{0}^{\prime}>0\right]} \\
& \vee\left[v_{2}^{\prime}<0\right] \wedge\left(\left[2 Z v_{2}^{\prime}+v_{1}^{\prime}<0\right] \vee\left[Z^{2} v_{2}^{\prime}+Z v_{1}^{\prime}+v_{0}^{\prime}>0\right]\right) \\
& \vee\left[v_{2}^{\prime}=0\right] \wedge \quad\left[v_{1}^{\prime}>0\right] \wedge \quad\left[Z v_{1}^{\prime}+v_{0}^{\prime}>0\right] \\
& {\left[z_{1}>Z\right] \Longleftrightarrow \quad\left[v_{2}^{\prime}>0\right] \wedge\left(\left[2 Z v_{2}^{\prime}+v_{1}^{\prime}<0\right] \vee\left[Z^{2} v_{2}^{\prime}+Z v_{1}^{\prime}+v_{0}^{\prime}<0\right]\right)} \\
& \vee\left[v_{2}^{\prime}<0\right] \wedge\left[2 Z v_{2}^{\prime}+v_{1}^{\prime}>0\right] \wedge\left[Z^{2} v_{2}^{\prime}+Z v_{1}^{\prime}+v_{0}^{\prime}<0\right] \\
& \vee\left[v_{2}^{\prime}=0\right] \wedge \quad\left[v_{1}^{\prime}>0\right] \wedge \quad\left[Z v_{1}^{\prime}+v_{0}^{\prime}<0\right] \\
& {\left[z_{2}<Z\right] \Longleftrightarrow \quad\left[v_{2}^{\prime}>0\right] \wedge\left(\left[2 Z v_{2}^{\prime}+v_{1}^{\prime}>0\right] \vee\left[Z^{2} v_{2}^{\prime}+Z v_{1}^{\prime}+v_{0}^{\prime}<0\right]\right)} \\
& \vee\left[v_{2}^{\prime}<0\right] \wedge\left[2 Z v_{2}^{\prime}+v_{1}^{\prime}<0\right] \wedge\left[Z^{2} v_{2}^{\prime}+Z v_{1}^{\prime}+v_{0}^{\prime}<0\right] \\
& \vee\left[v_{2}^{\prime}=0\right] \wedge \quad\left[v_{1}^{\prime}<0\right] \wedge \quad\left[Z v_{1}^{\prime}+v_{0}^{\prime}<0\right] \\
& {\left[z_{2}>Z\right] \Longleftrightarrow \quad\left[v_{2}^{\prime}>0\right] \wedge\left[2 Z v_{2}^{\prime}+v_{1}^{\prime}<0\right] \wedge\left[Z^{2} v_{2}^{\prime}+Z v_{1}^{\prime}+v_{0}^{\prime}>0\right]} \\
& \vee\left[v_{2}^{\prime}<0\right] \wedge\left(\left[2 Z v_{2}^{\prime}+v_{1}^{\prime}>0\right] \vee\left[Z^{2} v_{2}^{\prime}+Z v_{1}^{\prime}+v_{0}^{\prime}>0\right]\right) \\
& \vee\left[v_{2}^{\prime}=0\right] \wedge \quad\left[v_{1}^{\prime}<0\right] \wedge \quad\left[Z v_{1}^{\prime}+v_{0}^{\prime}>0\right]
\end{aligned}
$$

It holds:

$$
\begin{aligned}
& v_{2}^{\prime}=\frac{\alpha_{01}^{2}+\alpha_{02}^{2}}{\alpha_{03}^{2}}-\frac{\gamma_{01}^{2}+\gamma_{02}^{2}}{\gamma_{03}^{2}}, \\
& v_{1}^{\prime}=2 \frac{\alpha_{02} \alpha_{23}-\alpha_{01} \alpha_{31}}{\alpha_{03}^{2}}-2 \frac{\gamma_{02} \gamma_{23}-\gamma_{01} \gamma_{31}}{\gamma_{03}^{2}} \\
& v_{0}^{\prime}=\frac{\alpha_{23}^{2}+\alpha_{31}^{2}}{\alpha_{03}^{2}}-\frac{\gamma_{23}^{2}+\gamma_{31}^{2}}{\gamma_{03}^{2}} .
\end{aligned}
$$

The predicates $\left[v_{1}^{\prime 2}-4 v_{2}^{\prime} v_{0}^{\prime} \geq 0\right]$ and $\left[u_{1}^{\prime 2}-4 u_{2}^{\prime} u_{0}^{\prime} \geq 0\right]$ are equivalent, since both express the fact that the rotating line $L_{a b}$ collides with the stationary line $L_{c d}$ during a full rotation. On the basis of relations (11) and equation (3) it holds:

$$
u_{1}^{\prime 2}-4 u_{2}^{\prime} u_{0}^{\prime}=\alpha_{03}^{2} \gamma_{03}^{2} \cdot\left(v_{1}^{\prime 2}-4 v_{2}^{\prime} v_{0}^{\prime}\right) .
$$

According to equation (8) the predicate $\left[v_{1}^{\prime 2}-4 v_{2}^{\prime} v_{0}^{\prime} \geq 0\right]$ is linear in the expressions $\alpha_{01}^{2}+\alpha_{02}^{2}, \alpha_{23}^{2}+\alpha_{31}^{2}, \alpha_{01} \alpha_{31}-\alpha_{02} \alpha_{23}, \alpha_{03}^{2}$ and $\alpha_{12}^{2}$. 
Now let us consider the predicates $\left[v_{2}^{\prime} \lessgtr 0\right],\left[2 Z v_{2}^{\prime}+v_{1}^{\prime} \lessgtr 0\right]$ and $\left[Z^{2} v_{2}^{\prime}+Z v_{1}^{\prime}+v_{0}^{\prime} \lessgtr 0\right]$. A multiplication with $\alpha_{03}^{2} \gamma_{03}^{2}$ yields:

$$
\begin{aligned}
{\left[v_{2}^{\prime} \lessgtr 0\right] \Longleftrightarrow } & {\left[\gamma_{03}^{2}\left(\alpha_{01}^{2}+\alpha_{02}^{2}\right)-\left(\gamma_{01}^{2}+\gamma_{02}^{2}\right)\left(\alpha_{03}^{2}\right) \lessgtr 0\right], } \\
{\left[2 Z v_{2}^{\prime}+v_{1}^{\prime} \lessgtr 0\right] \Longleftrightarrow } & {\left[2 Z \gamma_{03}^{2}\left(\alpha_{01}^{2}+\alpha_{02}^{2}\right)-2 Z\left(\gamma_{01}^{2}+\gamma_{02}^{2}\right)\left(\alpha_{03}^{2}\right)\right.} \\
& \left.+2 \gamma_{03}^{2}\left(\alpha_{02} \alpha_{23}-\alpha_{01} \alpha_{31}\right)-2\left(\gamma_{02} \gamma_{23}-\gamma_{01} \gamma_{31}\right)\left(\alpha_{03}^{2}\right) \lessgtr 0\right], \\
{\left[Z^{2} v_{2}^{\prime}+Z v_{1}^{\prime}+v_{0}^{\prime} \lessgtr 0\right] \Longleftrightarrow } & {\left[Z^{2} \gamma_{03}^{2}\left(\alpha_{01}^{2}+\alpha_{02}^{2}\right)-Z^{2}\left(\gamma_{01}^{2}+\gamma_{02}^{2}\right)\left(\alpha_{03}^{2}\right)\right.} \\
& +2 Z \gamma_{03}^{2}\left(\alpha_{02} \alpha_{23}-\alpha_{01} \alpha_{31}\right)-\left(\gamma_{23}^{2}+\gamma_{31}^{2}\right)\left(\alpha_{03}^{2}\right) \\
& \left.-2 Z\left(\gamma_{02} \gamma_{23}-\gamma_{01} \gamma_{31}\right)\left(\alpha_{03}^{2}\right)+\gamma_{03}^{2}\left(\alpha_{23}^{2}+\alpha_{31}^{2}\right) \lessgtr 0\right] .
\end{aligned}
$$

If $Z=c_{3}, d_{3}$ (see condition 10 ), then these predicates are linear in the four expressions $\alpha_{01}^{2}+\alpha_{02}^{2}, \alpha_{03}^{2}, \alpha_{02} \alpha_{23}-\alpha_{01} \alpha_{31}, \alpha_{23}^{2}+\alpha_{31}^{2}$, and the corresponding coefficients only depend on the coordinates of the points $\mathbf{c}$ and $\mathbf{d}$.

However if $Z=a_{3}, b_{3}$, then one can define the expressions $Z^{k}\left(\alpha_{01}^{2}+\alpha_{02}^{2}\right), Z^{k} \alpha_{03}^{2}$, $Z^{k}\left(\alpha_{02} \alpha_{23}-\alpha_{01} \alpha_{31}\right), Z^{k}\left(\alpha_{23}^{2}+\alpha_{31}^{2}\right)$ for $k=0,1,2$, so that the former predicates are linear in at most six of these expressions, where again the corresponding coefficients only depend on the coordinates of the points $\mathbf{c}$ and $\mathbf{d}$.

\subsection{Linearization}

To summarize we firstly computed the conditions for the fact that the moving line $L_{a b}$ intersects the stationary line $L_{c d}$ during a time interval $[0, t]$. In the next step (see sections 4.4 and 4.7 ) we got the additional conditions for the intersection of the corresponding line segments. The combination of these two sets of conditions gives the wanted linearization, i.e.

$$
\left[\exists \tau: 0<\tau<t, l_{a b}(\tau) \cap l_{c d} \neq \emptyset\right] \Longleftrightarrow \bigvee_{i}\left(\left[0<\tau_{i}<t\right] \wedge\left[l_{a b}\left(\tau_{i}\right) \cap l_{c d} \neq \emptyset\right]\right)
$$

Until now we have found linearizations with $\operatorname{dim}=6$ where $\delta_{k}^{i j}(\mathbf{c}, \mathbf{d})$ are polynomials in the coordinates of $\mathbf{c}$ and $\mathbf{d}$ and $\zeta_{k}^{i j}(\mathbf{a}, \mathbf{b}, t)$ are polynomials in $t$ and the coordinates of $\mathbf{a}$ and $\mathbf{b}$. In order to reduce the dimension we divide each inequality by a positive coefficient $\zeta_{k}^{i j}(\mathbf{a}, \mathbf{b}, t)$, which we can always find using the fact that $\alpha_{03}>0$. So we get a linearization with dimension $\operatorname{dim}=5$. For example let us consider the condition $\left[2 t u_{2}^{\prime}+u_{1}^{\prime}>0\right]$, where

$$
\begin{aligned}
2 t u_{2}^{\prime}+u_{1}^{\prime}= & 2 \gamma_{01}\left(-t \alpha_{23}+\alpha_{31}\right)+2 \gamma_{02}\left(-t \alpha_{31}-\alpha_{23}\right)+2 \gamma_{03}\left(t \alpha_{12}\right) \\
& +2 \gamma_{12}\left(t \alpha_{03}\right)+2 \gamma_{23}\left(-t \alpha_{01}+\alpha_{02}\right)+2 \gamma_{31}\left(-t \alpha_{02}-\alpha_{01}\right) .
\end{aligned}
$$

We can divide the inequality by the term $t \alpha_{03}>0$ and replace the inequality $2 t u_{2}^{\prime}+u_{1}^{\prime}>0$ by

$$
\begin{aligned}
0< & 2 \gamma_{01} \frac{\left(-t \alpha_{23}+\alpha_{31}\right)}{t \alpha_{03}}+2 \gamma_{02} \frac{\left(-t \alpha_{31}-\alpha_{23}\right)}{t \alpha_{03}}+2 \gamma_{03} \frac{t \alpha_{12}}{t \alpha_{03}} \\
& \left.+2 \gamma_{23} \frac{\left(-t \alpha_{01}+\alpha_{02}\right)}{t \alpha_{03}}+2 \gamma_{31} \frac{\left(-t \alpha_{02}-\alpha_{01}\right)}{t \alpha_{03}}+2 \gamma_{12}\right) .
\end{aligned}
$$

Now we can apply the construction of section 3.2 , especially Corollary 3 . 


\section{Alternative solution for the translational case}

Let $L_{1}$ and $L_{2}$ be two sets of line segments in 3 -space, each consisting of $n$ elements. We translate the set $L_{1}$ in direction $v$ for the distance $t$. We consider the following problems (we assume that no line segment is moving in the direction of its supporting line: this case can be solved with an algorithm computing the intersections between a set of red line segments and a set of blue line segments.):

- For each $l \in L_{1}$ determine the time of the first collision with an element of $L_{2}$ respectively the first such line segment (if any exists),

- determine the first collision between any element of $L_{1}$ and $L_{2}$.

Clearly we can solve the second problem by finding the minimum solution of the first one.

We will proceed in two steps. Firstly we construct an algorithm for emptiness queries, i.e. given a moving query segment determine if there is any collision with $L_{2}$ and if there is exactly one collision compute it. Then we apply the parametric search technique of [MEG83] for computing the first such hit segment of $L_{2}$.

In the following we reduce the emptiness problem to a static problem and solve it with known range searching techniques.

During its movement a segment moves over a quadrilateral which all segments of $L_{2}$, that are hit during the translation, have to intersect. We can triangulate the quadrilateral and determine all segments intersecting one of the resulting triangles. During this process it can happen that we count segments twice, if they intersect the common edge of the two triangles. To avoid this we can filter them out using a data structure for computing the intersection between a given set of segments and a query segment. But for our emptiness problem it is enough to work inaccurately. We can count the number of collisions and when there are less than three of them we compute them and check whether they are different.

Therefore we have to consider the following subproblem:

Given a set $L_{2}$ of $n$ line segments, construct a data structure such that for any query triangle $t$ one can efficiently count/compute the incidences with $L_{2}$.

In [PEL92] Pellegrini describes the query triangle as the intersection of three half-planes and gives a sketch of a solution.

We construct a multilevel data structure. In the first level we determine the line segments of $L_{2}$ which intersect the plane aff $(t)$. This can be done using a point location structure for locating the dual point of aff $(t)$ in the arrangement of $n$ spatial double wedges which are dual to the segments in $L_{2}$. (Another way of solving this intersection problem could be a two-level halfspace range searching structure which first computes the subset $S_{2}^{\prime}$ of 
all segments with left endpoints on one side of aff $(t)$ and then determine those of them with right endpoint on the other side of aff $(t)$ ).

When we have computed the segments intersecting aff $(t)$, we only have to consider their supporting lines (which does not change the incidences with $t$ ). In the following levels we use three times a data structure for determining incidences between a set of lines and a query halfplane in $\mathbb{R}^{3}$. We use [AM92] and get

Theorem 5 Given $n$ segments there is a data structure using $O(m)$ space, $n^{1+\epsilon} \leq m \leq$ $n^{4+\epsilon}$, such that we can count the number of segments intersected by a query triangle in time $O\left(n^{1+\epsilon} / m^{1 / 4}\right)$. For reporting these $k$ segments we need $O(k)$ additional steps. The structure can be build in time $O(m)$.

The query algorithm can be replaced by a parallel version running in $O$ (polylog $(n)$ ) parallel steps using $O\left(n^{1+\epsilon} / m^{1 / 4}\right)$ processors, which allows us to apply the parametric search technique efficiently.

Using this technique we get the following result.

Theorem 6 Given a set $S$ of n line segments in 3-space. We can preprocess it in time $O(m), n^{1+\epsilon} \leq m \leq n^{4+\epsilon}$, using $O(m)$ space, such that for an arbitrary line segment $l$ and an arbitrary direction $v$ we can determine the first collision of $l$ (moving in dirction $v$ ) with an element of $S$ in time $O\left(n^{1+\epsilon} / m^{1 / 4}\right)$.

Remark 7 In a similar way we can apply an algorithm for computing intersections between a set of triangles and a query segment (see [PEL93]) for answering collision queries between a set of moving segments and a (stationary) query segment with the same bounds.

Corollary 8 Given a set of $n$ line segments, each of which is moving translationally in the direction $v$ over distance $t$ and a set of $n$ stationary line segments in $\mathbb{R}^{3}$ we can compute the first collision between the two sets in time $O\left(n^{8 / 5+\epsilon}\right)$.

\section{Collisions between facets and vertices}

Recall that we consider two polyhedra one of which is moving (let us say $P_{1}$ ) whereas the other one, $P_{2}$, is stationary. Let $V_{i}, E_{i}, F_{i}, i=1,2$, denote the sets of vertices, edges and facets of the polyhedra. Only translations or rotations are permitted as motions (w.l.o.g. we assume that an axis of rotation has to intersect the center of the coordinate-system). Until now we have shown how to compute the first collision between the edges of $P_{1}$ and $P_{2}$. We still have to determine the first facet of $P_{2}$ hit by a vertex of $P_{1}$ respectively the first facet of the moving polyhedron which collides with a vertex of $P_{2}$. A solution to this problem is already presented in [ScH94] based on ideas from [Nu 85]. The facets and vertices are projected into a 2-dimensional space and a plane sweep technique is applied. 
For completeness we present a sketch of his construction. We only determine the time of the first collision between the set of vertices of $P_{1}$ and the set of faces of $P_{2}$.

In case of a translation we project the facets and vertices onto a plane perpendicular to the direction of the motion. If the polyhedron $P_{1}$ is rotating about an axis (intersecting the coordinate-center) we can apply a similar method, but we have to work with cylindrical coordinates: the projection is done by removing the angle-component. In both cases we get in the projection-plane a point set $\overline{V_{1}}$ which is the image of the vertices of $P_{1}$ and 2-dimensional regions $\overline{F_{2}}$ bounded by line segments respectively hyperbola segments. Now we execute a plane sweep: stop-points/halts are starting- and end-points, extremaland intersection-points of the segments. Between two consecutive halts the ordering of the intersection-points between the segments and the plane sweep $S$ is always the same. Therefore we can save the active segments in a balanced search tree which will be the primary structure for saving more informations.

Let $R$ be a region between two segments, which are adjacent on $S$, and assume that $S$ paints over $R$ between two consecutive halts. Every vertex of $P_{1}$, whose projection $\bar{v}$ lies in $R$, can only collide with faces of $P_{2}$, the projections $\bar{f}$ of which contain $R$. Therefore for each region $R$ we keep track of the set $F_{R}=\left\{f \in F_{2} \mid R \subseteq \bar{f}\right\}$. Thus for every segment we save the set $F_{R}$ of the region lying above it.

Dependent on the kind of motion we can define an ordering for each set $F_{R}$. During a rotation every point with projection in $R$ will stab the regions in $F_{R}$ with the same cyclic ordering; in the case of a translation we will get a linear order. As secondary structure which stores the elements in $F_{R}$ we again use a balanced search tree which allows to find the first facet hit by a vertex projected onto $R$ in logarithmic time.

The sweep line stops at every point $\bar{v} \in \bar{V}$. There we determine the region $R$ containing $\bar{v}$ and search the facet of $F_{R}$ which is hit by the corresponding vertex first. Both steps can be done in logarithmic time using the tree structure.

During the sweep we have to hold all regions intersected by the sweep plane $S$ as well as the sets $F_{R}$. To save space we only store the changes of the sets $F_{R}$ (see [Nu85]). Using this idea each set $F_{R}$ can be stored with logarithmical costs.

The run time of the algorithm is $O\left(\left(\left|V_{1}\right|+\left|E_{2}\right|+C_{\overline{E_{2}}}\right) \log \left|E_{2}\right|\right)$ where $C_{\overline{E_{2}}}$ denotes the number of intersections of the projected edges of $P_{2}$. Unfortunately this value could be quadratic in the complexity of the polyhedron. Therefore we divide the problem into several smaller subproblems. W.l.o.g. we assume that the facets of $P_{2}$ are triangles (a triangulation of the surface does not change the asymptotic complexity of $P_{2}$ ). We divide $F_{2}$ in $\sqrt{\left|F_{2}\right|}$ many subsets of size $\sqrt{\left|F_{2}\right|}$. For each subset we execute the above plane sweep algorithm.

Theorem 9 Consider two polyhedra $P_{1}, P_{2}$, one of which is moving translationally or rotating about a fixed axis. The first collision between a vertex of one of them and a facet of the other can be computed in time $O\left(\left(\left|P_{1}\right|+\left|P_{2}\right|\right)^{3 / 2} \log \left(\left|P_{1}\right|+\left|P_{2}\right|\right)\right)$. 


\section{Conclusion}

We have shown how to determine the collision between a stationary and a moving polyhedron in sub-quadratic time. For that we have computed the first collision between vertices of one polyhedron and facets of the other and the first collision between the edges of the polyhedra. We have reduced the latter task to the formulation of an appropriate linearization which is derived by explicit computation of the collision times. We could do this because the equations of the motions have degree at most two. The natural question is how we can proceed if the motion of the polyhedron is more complicated, i.e. if the equations have degree greater than five (then no explicit formulation of the roots exists).

\section{A Appendix}

\section{A.1 Roots of a quadratic equation}

$$
\begin{aligned}
& q(x)=a x^{2}+b x+c=0, \quad a \neq 0 \\
& x_{1}=\frac{-b+\sqrt{b^{2}-4 a c}}{2 a} \\
& x_{2}=\frac{-b-\sqrt{b^{2}-4 a c}}{2 a}
\end{aligned}
$$

The quadratic equation $q(x)$ has real roots, iff the discriminant satisfies the condition $\left[b^{2}-4 a c \geq 0\right]$.

Conditions of the form $\left[x_{i}<X\right]$ or $\left[x_{i}>X\right]$ can be transformed so that they are linear in the coefficients of $a, b$ and $c$. As an example we consider the condition $\left[x_{1}<X\right]$.

1. case: $[a>0]$

$$
\begin{aligned}
& x_{1}=\frac{-b+\sqrt{b^{2}-4 a c}}{2 a}<X \\
\Longleftrightarrow & \sqrt{b^{2}-4 a c}<2 X a+b, \quad \text { since }[a>0] \\
\Longleftrightarrow & {[2 X a+b>0] \wedge\left[b^{2}-4 a c<(2 X a+b)^{2}\right] } \\
& b^{2}-4 a c<(2 X a+b)^{2} \\
\Longleftrightarrow & 4 a\left(X^{2} a+X b+c\right)>0 \\
\Longleftrightarrow & X^{2} a+X b+c>0, \quad \text { since }[a>0]
\end{aligned}
$$

As a consequence we have

$$
\begin{aligned}
& {[a>0] \wedge\left[x_{1}<X\right] } \\
\Longleftrightarrow \quad & {[a>0] \wedge[2 X a+b>0] \wedge\left[X^{2} a+X b+c>0\right] }
\end{aligned}
$$


2. case: $[a<0]$

$$
\begin{aligned}
& x_{1}=\frac{-b+\sqrt{b^{2}-4 a c}}{2 a}<X \\
\Longleftrightarrow & \sqrt{b^{2}-4 a c}>2 X a+b, \quad \text { since }[a<0] \\
\Longleftrightarrow & {[2 X a+b<0] \vee\left[b^{2}-4 a c>(2 X a+b)^{2}\right] } \\
& b^{2}-4 a c>(2 X a+b)^{2} \\
\Longleftrightarrow & 4 a\left(X^{2} a+X b+c\right)<0 \\
\Longleftrightarrow & X^{2} a+X b+c>0 \quad \text { since }[a<0]
\end{aligned}
$$

Therefore the following holds:

$$
\begin{aligned}
& {[a<0] \wedge\left[x_{1}<X\right] } \\
& \Longleftrightarrow \quad[a<0] \wedge\left([2 X a+b<0] \vee\left[X^{2} a+X b+c>0\right]\right)
\end{aligned}
$$

3. case: $[a=0]$

If the leading coefficient of the quadratic equation vanishes we have to deal with a linear equation. Its root can be obtained by

$$
\lim _{a \rightarrow 0} x_{1}=\left\{\begin{array}{cc}
-c / b & \text { for } \quad b>0 \\
\pm \infty & \text { for } b<0
\end{array}, \quad \lim _{a \rightarrow 0} x_{2}=\left\{\begin{array}{cc} 
\pm \infty & \text { for } b>0 \\
-c / b & \text { for } b<0
\end{array} .\right.\right.
$$

Thus we get

$$
\begin{aligned}
& {[a=0] \wedge\left[x_{1}<X\right] } \\
& \Longleftrightarrow \quad[a=0] \wedge[b>0] \wedge[X b+c>0]
\end{aligned}
$$

In the same way one can linearize the conditions $\left[x_{1}>X\right],\left[x_{2}<X\right]$ and $\left[x_{2}>X\right]$. If we assume that $\left[b^{2}-4 a c \geq 0\right]$ the following holds:

$$
\begin{array}{rlrl}
{\left[x_{1}<X\right] \Longleftrightarrow \quad} & {[a>0] \wedge[2 X a+b>0] \wedge\left[X^{2} a+X b+c>0\right]} \\
& {[a<0] \wedge\left([2 X a+b<0] \vee\left[X^{2} a+X b+c>0\right]\right)} \\
& {[a=0] \wedge \quad[b>0] \wedge} & {[X b+c>0]} \\
{\left[x_{1}>X\right] \Longleftrightarrow \quad[a>0] \wedge\left([2 X a+b<0] \vee\left[X^{2} a+X b+c<0\right]\right)} \\
& {[a<0] \wedge[2 X a+b>0] \wedge\left[X^{2} a+X b+c<0\right]} \\
& {[a=0] \wedge \quad[b>0] \wedge} & {[X b+c<0]} \\
{\left[x_{2}<X\right] \Longleftrightarrow \quad[a>0] \wedge\left([2 X a+b>0] \vee\left[X^{2} a+X b+c<0\right]\right)} \\
& {[a<0] \wedge[2 X a+b<0] \wedge\left[X^{2} a+X b+c<0\right]} \\
& {[a=0] \wedge} & {[b<0] \wedge} & {[X b+c<0]} \\
{\left[x_{2}>X\right] \Longleftrightarrow \quad} & {[a>0] \wedge[2 X a+b<0] \wedge\left[X^{2} a+X b+c>0\right]} \\
& {[a<0] \wedge\left([2 X a+b>0] \vee\left[X^{2} a+X b+c>0\right]\right)} \\
& {[a=0] \wedge \quad[b<0] \wedge} & {[X b+c>0]}
\end{array}
$$




\section{A.2 Degeneracies}

\section{A.2.1 Translation}

The collision times $\tau_{0}$ of the translational moving line $L_{a b}$ and the stationary line $L_{c d}$ satisfy the equality (4), i.e.

$$
u_{1} \tau+u_{0}=0
$$

Here we consider the case $u_{1}=0$, which means that a collision during the translation can only occur if $u_{1}=0$ and $\bar{L}_{a b}=\bar{L}_{c d}$.

These conditions describe the following situation: the lines $L_{a b}$ and $L_{c d}$ have to be parallel or to intersect; additionally they must lie in the same plane perpendicular to the $x_{1} x_{2^{-}}$ plane. In this case the collision detection of the line segments can be described as a two-dimensional problem.

It is easy to see that a collision between two segments in 2-space is always a collision between a vertex of one segment and the other segment. We will only demonstrate how to test whether the point a collides with the segment $l_{c d}$, the other cases being similar. The collision occurs iff there are $\mu, 0 \leq \mu \leq 1$, and $\lambda, 0<\lambda<t$, such that

$$
\lambda \mathbf{e}_{3}+\mu(\mathbf{c}-\mathbf{d})=\mathbf{a}-\mathbf{c},
$$

For $d_{2} \neq c_{2}$ this equation is satisfied iff

$$
\begin{gathered}
\left(a_{2}-c_{2}\right)\left(d_{1}-c_{1}\right)=\left(a_{1}-c_{1}\right)\left(d_{2}-c_{2}\right) \wedge \\
0 \leq \frac{a_{2}-c_{2}}{d_{2}-c_{2}} \leq 1 \wedge \\
0<c_{3}-a_{3}+\frac{a_{2}-c_{2}}{d_{2}-c_{2}}\left(d_{3}-c_{3}\right)<t .
\end{gathered}
$$

Further case decompositions (e.g. $d_{3}>c_{3}, d_{3}=c_{3}$ ) yield a linearization of dimension less than 5 .

\section{A.2.2 Rotation}

The collision times $\tau_{i}$ of the rotating line $L_{a b}$ and the stationary line $L_{c d}$ satisfy the equation (7) i.e.

$$
u_{2}^{\prime} \tau^{2}+u_{1}^{\prime} \tau+u_{0}^{\prime}=0 .
$$

Here we consider the case $u_{2}^{\prime}=u_{1}^{\prime}=0$, which means that a collision during the rotation can only occur if $u_{0}^{\prime}=0$ and the lines lie on the same cone respectively cylinder. Therefore collision detection can be reduced to a 2-dimensional problem, so that we only need to test a collision between a vertices and segments. The same holds for the degeneracy of equation (9) which means that the lines lie on the same hyperboloid. 


\section{A.3 Correspondence between collision times and collision points}

Given: Two lines $L_{a b}$ and $L_{c d}$. Let $L_{a b}(\tau)$ rotate about the $x_{3}$-axis, and assume that $L_{a b}\left(\tau_{i}\right) \cap L_{c d} \neq \emptyset$ holds at time $\tau_{1}$ and $\tau_{2}$.

Wanted: The $x_{3}$-coordinate of the intersection point between $L_{a b}\left(\tau_{i}\right)$ and $L_{c d}$.

For the $x_{3}$-coordinate of the intersection point of two lines $L_{a b}^{\prime}$ and $L_{c d}$ it holds:

$$
z_{i}=\frac{\alpha_{03}^{\prime} \gamma_{12}+\alpha_{23}^{\prime} \gamma_{01}+\alpha_{31}^{\prime} \gamma_{02}}{\alpha_{01}^{\prime} \gamma_{02}-\alpha_{02}^{\prime} \gamma_{01}}
$$

We substitute $L_{a b}\left(\tau_{i}\right)$ for $L_{a b}^{\prime}$ :

$$
\begin{aligned}
& \alpha_{01}^{\prime}=\frac{1-\tau_{i}^{2}}{1+\tau_{i}^{2}} \alpha_{01}+\frac{2 \tau_{i}}{1+\tau_{i}^{2}} \alpha_{02}, \quad \alpha_{02}^{\prime}=-\frac{2 \tau_{i}}{1+\tau_{i}^{2}} \alpha_{01}+\frac{1-\tau_{i}^{2}}{1+\tau_{i}^{2}} \alpha_{02} \\
& \alpha_{03}^{\prime}=\alpha_{03}, \quad \alpha_{12}^{\prime}=\alpha_{12} \\
& \alpha_{23}^{\prime}=\frac{1-\tau_{i}^{2}}{1+\tau_{i}^{2}} \alpha_{23}+\frac{2 \tau_{i}}{1+\tau_{i}^{2}} \alpha_{31}, \quad \alpha_{31}^{\prime}=-\frac{2 \tau_{i}}{1+\tau_{i}^{2}} \alpha_{23}+\frac{1-\tau_{i}^{2}}{1+\tau_{i}^{2}} \alpha_{31}, \quad \text { where } \\
& \tau_{i}=\frac{-u_{1}^{\prime} \pm \sqrt{u_{1}^{\prime 2}-4 u_{2}^{\prime} u_{0}^{\prime}}}{2 u_{2}^{\prime}}, \quad \text { and } \\
& u_{2}^{\prime}=-\gamma_{23} \alpha_{01}-\gamma_{31} \alpha_{02}+\gamma_{12} \alpha_{03}+\gamma_{03} \alpha_{12}-\gamma_{01} \alpha_{23}-\gamma_{02} \alpha_{31} \\
& u_{1}^{\prime}=-2 \gamma_{31} \alpha_{01}+2 \gamma_{23} \alpha_{02}-2 \gamma_{02} \alpha_{23}+2 \gamma_{01} \alpha_{31} \\
& u_{0}^{\prime}=\gamma_{23} \alpha_{01}+\gamma_{31} \alpha_{02}+\gamma_{12} \alpha_{03}+\gamma_{03} \alpha_{12}+\gamma_{01} \alpha_{23}+\gamma_{02} \alpha_{31}
\end{aligned}
$$

Inserting the Pliicker coordinates $\alpha_{i j}^{\prime}$ into equation (14) results in

$$
\begin{gathered}
z_{i}=\frac{ \pm A x+B}{ \pm C x+D}=\frac{A C x^{2}-B D \pm(B C-A D) x}{C^{2} x^{2}-D^{2}} \\
\text { where } \quad x=\frac{1}{2} \sqrt{u_{1}^{\prime 2}-4 u_{2}^{\prime} u_{0}^{\prime}}
\end{gathered}
$$

Thereby it holds:

$$
\begin{aligned}
B C-A D= & \gamma_{03} \alpha_{03}\left(\gamma_{12}^{2} \alpha_{01}^{2}+\gamma_{12}^{2} \alpha_{02}^{2}-\gamma_{01}^{2} \alpha_{12}^{2}-\gamma_{02}^{2} \alpha_{12}^{2}\right) \\
& \left(\gamma_{23} \alpha_{01}+\gamma_{31} \alpha_{02}-\gamma_{12} \alpha_{03}-\gamma_{03} \alpha_{12}+\gamma_{01} \alpha_{23}+\gamma_{02} \alpha_{31}\right)^{2} \\
C^{2} x^{2}-D^{2}= & \left(\gamma_{12}^{2} \alpha_{01}^{2}+\gamma_{12}^{2} \alpha_{02}^{2}-\gamma_{01}^{2} \alpha_{12}^{2}-\gamma_{02}^{2} \alpha_{12}^{2}\right) \\
& \left(\gamma_{23} \alpha_{01}+\gamma_{31} \alpha_{02}-\gamma_{12} \alpha_{03}-\gamma_{03} \alpha_{12}+\gamma_{01} \alpha_{23}+\gamma_{02} \alpha_{31}\right)^{2} \\
& \left(\gamma_{03}^{2}\left(\alpha_{01}^{2}+\alpha_{02}^{2}\right)-\left(\gamma_{01}^{2}+\gamma_{02}^{2}\right) \alpha_{03}^{2}\right) \\
A C x^{2}-B D= & \left(\gamma_{12}^{2} \alpha_{01}^{2}+\gamma_{12}^{2} \alpha_{02}^{2}-\gamma_{01}^{2} \alpha_{12}^{2}-\gamma_{02}^{2} \alpha_{12}^{2}\right) \\
& \left(\gamma_{23} \alpha_{01}+\gamma_{31} \alpha_{02}-\gamma_{12} \alpha_{03}-\gamma_{03} \alpha_{12}+\gamma_{01} \alpha_{23}+\gamma_{02} \alpha_{31}\right)^{2} \\
& \left(\left(\gamma_{02} \gamma_{23}-\gamma_{01} \gamma_{31}\right) \alpha_{03}^{2}-\gamma_{03}^{2}\left(\alpha_{02} \alpha_{23}-\alpha_{01} \alpha_{31}\right)\right)
\end{aligned}
$$

Under the assumption that $\alpha_{03} \gamma_{03}>0$ applying equation (12) shows, that the $x_{3}-$ coordinate $z_{1}$ corresponds to $\tau_{1}$ and $z_{2}$ to $\tau_{2}$, as specified in equation 10 . 


\section{References}

[AM92] P. K. Agarwal, J. Mato1šsek: On range searching with semialgebraic sets, Proc. 17th Symp. on Math. Foundation of CS: 1-13 (1992)

[AM92B] P. K. Agarwal, J. Matoušek: Ray shooting and parametric search, Proc. 24th STOC: $517-526(1992)$

[Bo79] J. W. Boyse: Interference detection among solids and surfaces, CACM Vol. 22(1) (1979), S. 3-9

[CA84] J. Canny: On detecting collision between polyhedra, Proc. ECAI (1984), S. 533-542

[DK85] D. Dobkin, D. Kirkpatrick: A linear algorithm for determing the seperation of convex polyhedra, J. Algor. 6 (1985), S. 381-392

[DK90] D. Dobkin, D. Kirkpatrick: Determining the seperation of preprocessed polyhedra - a unified approach, Lecture Notes in Computer Science 443 (1990), S. 400-413

[DHKS90] D. Dobkin, J. Hershberger, D. Kirkpatrick, S. Suri: Implicitily searching convolutions and computing depth of collisions, Lecture Notes in Computer Science 450 (1990), S. 165-180

[Krev92] Marc van Kreveld: New Results on Data Structures in Computational Geometry, Ph.D. Thesis, University of Utrecht, The Netherlands, (1992)

[Mat93] J. Mato1šsek: Range searching with efficient hierarchical cuttings, Discrete Comput. Geom 10:159-182 (1993)

[Meg83] N. Megiddo. Applying parallel computation algorithms in the design of serial algorithms. Journal of the ACM 30: 852-865 (1983).

[Nu85] O. Nurmi: A fast algorithm for hidden-line elimination, BIT, Vol. 25 (1985), S. $466-472$

[PEL92] M. Pellegrini Incidence and nearest-neighbor problems for lines in 3-space, Proc. 8th Annu. ACM Symos. Comput. Geom.: 130-137 (1992)

[PeL93] M. Pellegrini: Ray shooting on triangles in 3-space, Algorithmica 9: 471-494 (1993)

[PS88] F. P. Preparata and M. I. Shamos: Computational Geometry: an Introduction, Springer-Verlag, New York, (1988)

[Sch94] Elmar Schömer: Interaktive Montagesimulation mit Kollisionserkennung, Ph.D. Thesis, Universität des Saarlandes, Germany, (1994) 